\title{
Education and Consumer Health Informatics
}

\author{
J. Mantas \\ Health Informatics Lab, University of Athens, Greece
}

Summary
Objectives: To report on recent efforts in the field of consumer health
informatics (CHI) and education.
Methods: A review of the literature we selected on using search
engines and Medline with terms from consumer health informatics
and education. Twenty two articles match these criteria.
Results: A great diversity of work spans the field of CHI. This relatively
new field now faces challenges due to rapidly advancing
technologies and the increasing empowerment of citizens connected
over the worldwide web.
Conclusion: The benefits of enhancing ClH components within
existing medical curricula are already being noted.. Additionally,
updated recommendations on health and medical informatics
should incorporate CHI.
Keywords
Health informatics, medical informatics, consumer health
informatics, education
Geissbuhler A, Haux R, KulikowskiC, editors. IMIA Yearbook of
Medical Informatics2007. Methods InfMed 2007 ; 46 Suppl I: $90-4$

\section{Introduction}

Health informatics has been rapidly changing over the past few years. Advances in bioinformatics and its integration into more traditional medical informatics is not only a challenge but also represents a huge potential for the emerging field of biomedical informatics $[1,2]$. After decades of development of information systems designed primarily for physicians and other healthcare managers and professionals, there is an increasing interest in reaching consumers and patients directly through computers and telecommunications systems. In the literature, consumer health informatics (CHI) is referred to as a discipline within health informatics that analyses consumers' needs for information, studies and implements methods of making information accessible to consumers, and models and integrates consumers' preferences into medical information systems. The field of CHI receives feedback from other disciplines such as medical informatics, nursing informatics, public health, health promotion, health education, marketing, and communication science. $\mathrm{CHI}$ is becoming one of the most challenging, rapidly expanding field in medical informatics making the patient and citizen the centre of attraction of new age of information and technology in health care. Furthermore the traditional educational curricula of health informatics are being modified to incorporate aspects of $\mathrm{CHI}$.

\section{Method}

This review is by no means exhaustive. It focuses on topics in the field of education and $\mathrm{CHI}$, given the markedly increased interest in this field. It is based on peer-reviewed journals, and its main source is Medline. It used the terms "consumer health informatics" and "education". The term health was replaced with the term medical to make sure that it would pick up earlier material also. Google Scholar was used for locating additional reports from nonindexed sources.

\section{Results}

A review of selected articles [3] demonstrates examples of excellent research in $\mathrm{CHI}$ and medical education. The methods presented can contribute to the development of systems for the education of both laypersons and health professionals.

Moehr, in [4], explores whether education in health/medical informatics should continue to evolve along the lines pursued since the early seventies, or whether a change is advisable. Origins and typical characteristics of European and US American approaches to health informatics education are identified. In Europe, holistic approaches based on a synthesis of medicine and informatics, that is, computer science with programs ranging from vocational training through university 
programs to doctoral and postdoctoral programs were characteristic. The US American approaches emphasized higher levels of education and a diverse selection of specialized subjects. Changes in health and health informatics are summarised. Two types of changes are identified: high-tech applications arising at the interface of imaging, robotics, and the -omics (genomics, proteomics, metabolomics), invasive applications focusing on consumer health informatics, and a move from curative to preventive health care. It is proposed that curative medicine is adequately served by current educational approaches, but that the move towards preventive health care requires a move towards education and change management for health professionals and health informatics professionals. Deda, in [5], indicates that science must have a common language. For centuries, Latin language carried out this job, but the progress in computer technology and the world of the internet over the past 20 years has begun to produce a new language with the new century; a computer language. The major information users, who need data language standardization, are the following: digital libraries and medical education systems, consumer health informatics facilities, medical education systems, World Wide Web applications, database systems, medical language processing, automatic indexing systems, image processing units, telemedicine, and new generation internet.

Gustafson et al. [6] reviews research and development related to the consumer health informatics system CHESS (The Comprehensive Health Enhancement Support System) by the Center for Health Systems Research and Analysis at the University of Wisconsin. The review places particular emphasis on what has been found with regards to the acceptance and use of such systems by high risk and underserved groups.

Mohr, in [7], summarizes the insights gained in collaborative research in a Canadian Network of Centres of Excellence, devoted to the promotion of evidence-based practice, and relates this experience to Internet support of health promotion and consumer health informatics. A subjective review of insights is involved. Work directed to the development of systems incorporating guidelines, care maps, etc., for use by professionals met with limited acceptance. Evidence-based tools for health care consumers are a desirable complement but require radically different content and delivery modes. In addition to evidence-based material offered by professionals, a wide array of Internet-based products and services provided by consumers for consumers emerged and is claimed to have shown its usefulness. The consumer-driven products and services provided via the Internet are a potentially important and beneficial complement to traditional health services. They affect health consumer-provider roles and require changes in healthcare practices.

In [8] it is emphasized that $\mathrm{CHI}$ means different things to patients, health professionals, and health care systems. A broader perspective on this new and rapidly developing field enhances understanding and helps to better apply its methods and software.. This article provides an overview of CHI discussing its evolution and driving forces, along with applications to Personal Health Records, Internet transmission of personal health data, clinical e-mail, online pharmacies, and shared decisionmaking tools. Consumer Health Informatics is expected to become integrated with medical care, electronic medical records, and patient education, and impact the whole process and business of health care.
In [9] it is noted that although interest in $\mathrm{CHI}$ has increased, a consensus definition of $\mathrm{CHI}$ does not yet exist. A hypothesis-generating survey of AMIA members regarding the definition and research agenda for $\mathrm{CHI}$ came from soliciting participation of AMIA members in an Internet-based survey focusing on these issues. One hundred thirtyfive AMIA members responded. Participants indicated a broad spectrum of topics important to $\mathrm{CHI}$ including "self-help for disease management" and "patient access to their own medical records." CHI research was felt to rely heavily on public health methods such as epidemiology and outcomes research, a paradigm shift from traditional medical informatics. Responses indicated a perceived lack of funding and need for further research in CHI. A working definition should emphasize the multidisciplinary nature of $\mathrm{CHI}$, include consumer input into CHI design, and focus on public health approaches to evaluation.

In [10] current access and barriers to health information for consumers is explored. It considers how computers and other developments in information technology are ushering in the era of $\mathrm{CHI}$, and their future potential Based on today's developments, where the public is already having unprecedented ability to access information and to participate actively in evidence-based health care it is suggested that $\mathrm{CHI}$ be viewed as a whole new academic discipline, one that should be devoted to the exploration of the new possibilities that informatics is creating for consumers in relation to health and health care issues.

In [11] an AMIA special track entitled "Consumer Informatics Supporting Patients as Co-Producers of Quality" was devoted to examining CHI. As a field, it is developing rapidly, with worldwide changes occurring in the 
organization and delivery of health care and in the traditional roles of patient and provider. This paper describes key themes of the track; implications of the growing area of consumer health informatics; and recommendations for informatics research, design, and policy. Major issues that emerged from the panels and discussions involved changes in roles of consumers and providers, support to a patient-provider-information technology partnership; virtual (as opposed to physical) structures for health care and health care information delivery, and health care as an integrated part of one's life. Pane lists and participants at the meeting developed recommendations for informatics research, design, and policy, with an overarching focus on how to support the patientprovider-information technology partnership to provide more patient-centred health care. They recommended that AMIA take an active leadership role in consumer health informatics. Specific recommendations were made concerning research, new patient record systems, provider support, information access and evaluation, and policy and regulation.

Reference [12], provides yet another description of $\mathrm{CHI}$ as it empowers the individual patient and the public at large. Data sources included: published articles, research studies, and government reports related to interactive health communication and CHI. The paper's conclusions include: that application of $\mathrm{CHI}$ can provide information to patients and the public, promote selfcare, enable informed decision-making, promote healthy behaviours, and promote peer information exchange and social support. Quality, research methodology, and accessibility need all be increased to ensure that $\mathrm{CHI}$ achieves its potential to improve a nation's health. With the advent of the internet, the profusion of consumer health-related web sites, online support groups, and electronic patient-centred communications present new challenges for clinical practice. Health care providers have important roles in helping their patients as well as the public locate, assess, and interpret health information.

In [13] the advances of health informatics in Canada over the last 50 years are briefly reviewed to reveal the pervasiveness of their applications in health and health care. The relations to research in health informatics and health are pointed out. From this perspective it is argued that the evolution of consumer health informatics in the last decade has had a profound impact on the practice of medicine, on patient-physician relations and, hence, on the requirements for medical education. The different access to information and how it is used in educational environments will also dramatically affect how curricula are structured both at undergraduate and postgraduate levels. The impact of health informatics on medical education is further elaborated, and the requirements on infrastructure in support of this education are detailed. This infrastructure goes beyond instructional laboratories and includes academic units for medical informatics and, most importantly perhaps, funding resources and adjudication capacity for health informatics research and their integration into the Canadian research organization and the new Canadian Institutes of Health Research.

Reference [14] points out how health informatics has much to offer community health care. Computer networks and telecommunications provide particular support that can enhance the collaboration among clinicians, care providers and patients. Special-purpose computer tools referred to as CHI represent the application of computer and information technologies specifically to support the health information and com- munication needs of patients and lay persons. Research projects like ComputerLink and CHESS demonstrate that $\mathrm{CHI}$ is acceptable to patients and promotes self-care and disease management. Three grand challenges must be faced to insure realization of the promise of health informatics to community health care: development of knowledge management and information discovery tools for patients, insurance of health information literacy for everyone, and re-engineering clinical practice to capitalize on patients as full partners in health care.

In [15] there is a discussion of how changes in reimbursement structures in health care have given rise to a rapidly growing focus on the consumer and this recent increase has been fuelled by the advent of the Web. CHI systems empower the consumer and aim to improve doctor-patient communication. The HouseCall system is presented as an example. The paper starts with a review how a consumer information system can be derived from an existing physician knowledge base (Iliad). Second, it presents evaluation studies that: 1) show how consumers are eager for non 'dumbed-down' content with easy access, 2) demonstrate the large spectrum of topics of interest and the 'natural' search strategies of health care consumers.

In [16] and [17] it is mentioned that lay health care consumers' emergence as active participants in online health care networks is a powerful new technological trend that promises to take the American health care system through all stages of cultural adaptation-substitution, innovation, and transformation. Soon everyone will recognize the fundamental changes online health has produced in the way we think and act about health care. A new subspecialty-consumer health informatics-covers two domains: (1) 
community CHI resources - the online networks, forums, databases, and World Wide Web sites that anyone with a home computer can access, and (2) clinical CHI resources-programs or systems developed by clinicians, system developers, or health maintenance organizations and provided to selected membership groups or patients. Sample communications among online selfhelpers within their chat groups, e-mail exchanges, bulletin boards, and USENET newsgroups for chosen health topics show what is on their minds, how they get information, how they use it, and how they correct it. Sometimes, clinicians have observed, lay care offers higher quality than professional care. Lay self-helpers, such as the originator of the Brain Tumour Mailing List, have observed several potential benefits in linking online self-helpers and providers and at a very modest cost. In a coming six-level system of information-age health care, patient-consumers may seek what they need in the following order: individual self-care, family and friends, informal self-help networks, the professional as coach, the professional as partner, and the professional as authority. Rapid developments in information technology offer better opportunities for consumer health information. Unfortunately, people with the greatest health burden often have the least access to health information and communication technologies. Multiple barriers contribute to disparities among different populations, including low literacy levels and language differences as well as economic, social, and cultural factors that may decrease access to technology. If Internet-based patient education is shown to improve health care outcomes, this may widen. In this workshop, participants will discuss common barriers to use of technology for disseminating electronic health information. In addition, poten- tial solutions to these problems will be explored, including a variety of interventions, such as: providing chronic disease patient education using touch screen computers in urban clinical waiting areas; providing training, education, and tools in accessing and evaluating on-line health information; and incorporating 'lay health advisors' to promote usage of consumer health information in the community. Additional example studies and implementation efforts targeting underserved or vulnerable populations will be presented and discussed. Ample time was to be made be available to review the interests and concerns of attendees in small and large group discussions.

\section{Discussion}

The field of health and medical informatics is rather new compared to more traditional disciplines such as physics, mathematics, medicine and other life sciences. The field itself is being updated due to new technologies and approaches as well as due to new innovations. Recently the decoding of the DNA and the human genome has added another sector into the traditional health and medical informatics. The after shocks of this new development are still being felt and are difficult to be absorbed across the field.

The difficulties in defining the continuous moving ahead field of health informatics makes it more difficult for educators in this field to define and update the curricula and competencies of the discipline. It is evident that one of the areas that newly came into the arena was consumer health informatics. The new field is challenging, is continuous updated, and involves not only the specialists in the field but also involves the public and citizens. The empowerment of patients in employing technological innovations and applications is becoming the main issue in health care in this century. Today, European countries and elsewhere are facing the challenge of delivering quality healthcare to all its citizens at affordable cost. Protracted medical care for an ageing society, the costs of managing chronic care diseases, and the increasing demand by consumers, clients, and citizens for best quality healthcare are major factors. Healthcare expenditure is already significant (in Europe $8.5 \%$ of the GNP on average) and rising faster than overall economic growth itself. The emerging situation calls for a change in the way the healthcare is delivered and the way medical and healthcare knowledge is managed and transferred in clinical practice. Health Informatics and Consumer Health Informatics are key to implement these changes in this information driven domain.

The field of Consumer Health Informatics as a highly interdisciplinary field aiming at: (a) improving productivity of healthcare systems by facilitating patient care at the point of need, health information processing and quicker transfer of knowledge to clinical practice; (b) continuous and more personalized care solutions, addressing the informed and responsible participation of patients and their informal carers in care processes, and responding to the needs of elderly people; (c) higher patient safety by optimising medical interventions and preventing errors.

All these activities should address consumer (user) needs, personal data security, confidentiality, privacy, as well as the reimbursement scheme and legal framework for using the new technologies.

The International Medical Informatics Association has issued ten years ago recommendations for health and medi- 
cal informatics education. Many educational efforts [18]-[25], [26] have been based on those recommendations. Recently, an effort in updating those recommendations has started. It is envisaged that in the upcoming recommendations the area of consumer health informatics will be included as part of the overall curriculum and competencies map of health and medical informatics.

\section{Conclusions}

Information and communication technology offer useful capabilities to improve illness prevention and safety of care, facilitating active participation of patients, consumers and enabling personalisation of care that open new opportunities for patient empowerment and health and disease management. The new capabilities of modelling, simulation, and biomedical information, combined with knowledge about diseases that ranges from the molecular to the organ and system levels could lead to a new predictive medicine [1] as well as new opportunities and facilities for the consumer of healthcare. This might help bring radical improvements to the quality and efficiency of our healthcare systems. To achieve this, educational innovations should parallel the research efforts in the field of health and medical informatics. Updating of curricula, training programmes and public awareness should be a continuous effort to the educators in this promising but challenging field.

\section{References}

1. Mantas J. Future trends in Health Informaticstheoretical and practical. Stud Health Technol Inform 2004;109:114-27.

2. Marschollek M. Advances in Education and Consumer Health Informatics. In: Geissbuhler A, Haux R, Kulikowski C, editors. IMIA Yearbook of Medical Informatics 2007. Methods Inf Med 2007;46 Suppl 1:95-7.

3. Marschollek M. Methods to meet the informational demands of patients and health professionals. Methods Inf Med 2006;45 Suppl 1:88-9.

4. Moehr JR. Where to in the next ten years of health informatics education? Methods Inf Med 2006;45 (3):283-7.

5. Deda H, Yakupoglu H. Communication in science. Acta Neurochir Suppl 2002;83:17-23.

6. Gustafson DH, Hawkins RP, Boberg EW, McTavish F, Owens B, Wise M, Berhe H, Pingree S. CHESS: ten years of research and development in consumer health informatics for broad populations, including the underserved. Medinfo.2001;10(Pt2):1459-563.

7. Moehr JR. Guidelines, the Internet, and personal health: insights from the Canadian HEALNet experience. Methods Inf Med 2002;41(3):230-4.

8. Klein-Fedyshin MS. Consumer Health Informatics - integrating patients, providers, and professionals online. Med Ref Serv Q 2002 Fall; 21(3):35-50.

9. Houston TK, Chang BL, Brown S, Kukafka R. Consumer health informatics: a consensus description and commentary from American Medical Informatics Association members. Proc AMIA Symp. 2001;:269-73.

10. Eysenbach G, Jadad AR. Evidence-based patient choice and consumer health informatics in the Internet age. J Med Internet Res 2001 Apr-Jun; 3(2):E19.

11. Kaplan B, Brennan PF. Consumer informatics supporting patients as co-producers of quality. J Am Med Inform Assoc 2001 Jul-Aug;8(4):309-16.

12. Houston TK, Ehrenberger HE. The potential of consumer health informatics. Semin Oncol Nurs 2001 Feb;17(1):41-7.

13. Moehr JR, Grant A. Medical informatics and medical education in Canada in the 21st century. Clin Invest Med $2000 \mathrm{Aug} ; 23(4): 275-80$

14. Brennan PF. Health informatics and community health: support for patients as collaborators in care. Methods Inf Med 1999 Dec;38(4-5):274-8.

15. Bouhaddou O, Lambert JG, Miller S. Consumer health informatics: knowledge engineering and evaluation studies of medical HouseCall. Proc AMIA Symp 1998;:612-6.

16. Ferguson T. Health online and the empowered medical consumer. Jt Comm J Qual Improv 1997 May;
23(5):251-7.

17. Ferguson T. Consumer health informatics. Health Forum J. 1995 Jan-Feb;38(1):28-33.

18. Mantas J, Diomidous M. Implementation and evaluation of the MSc course in health informatics in Greece.Methods Inf Med2007;46(1):90-2.

19. Jossif A, Pattichis CS, Kyriakides M, Pitsillides A, Kyriacou E, Dikaiakos M. Selected eHealth applications in Cyprus from the training perspective. Methods Inf Med. 2007;46(1):84-9.

20. Hersh WR. The full spectrum of biomedical informatics education at Oregon Health \& Science University. Methods Inf Med 2007;46(1):80-3.

21. Haux R, Knaup P, Leiner F. On educating about medical data management - the other side of the electronic health record. Methods Inf Med 2007;46(1):74-9.

22. Demiris G. Interdisciplinary innovations in biomedical and health informatics graduate education. Methods Inf Med 2007;46(1):63-6.

23. Mantas J. Health and medical informatics education. Methods Inf Med 2007;46(1):50-1.

24. Hasman A, Haux R. Modeling in biomedical informatics - an exploratory analysis (part 1). Methods Inf Med 2006;45(6):638-42.

25. Haux R, Ammenwerth E, Haber A, Hubner-Bloder G, Knaup-Gregori P, Lechleitner G, Leiner F, Weber R, Winter A, Wolff AC. Medical informatics education needs information system practicums in health care settings - experiences and lessons learned from 32 practicums at four universities in two countries. Methods Inf Med 2006;45(3):294-9.

26. Recommendations of the International Medica Informatics Association (IMIA) on education in health and medical informatics. Methods Inf Med 2000;39:267-77.

\section{Correspondence to:}

Prof. John Mantas.

Health Informatics Lab

University of Athens

123, Papadiamantopoulou Street, Goudi

GR-11527 Athens

Greece

Tel: + 30210746 1459/60

Fox: +302107461461

E-mail: jmantas@cc.voa.gr 